2016

\title{
Parts of the Whole: Teaching Quantitative Reasoning in an Exponential Decay Model
}

Dorothy Wallace

Dartmouth College, dorothy.wallace@dartmouth.edu

Follow this and additional works at: https://digitalcommons.usf.edu/numeracy

Part of the Higher Education Commons, and the Science and Mathematics Education Commons

\section{Recommended Citation}

Wallace, Dorothy. "Parts of the Whole: Teaching Quantitative Reasoning in an Exponential Decay Model." Numeracy 9, Iss. 2 (2016): Article 12. DOI: http://dx.doi.org/10.5038/1936-4660.9.2.12 


\title{
Parts of the Whole: Teaching Quantitative Reasoning in an Exponential Decay Model
}

\author{
Abstract \\ The simple calculus example of exponential decay can be an excellent vehicle for teaching quantitative \\ reasoning in calculus or differential equations. Insect maturation provides a rich context for thinking \\ about the meaning of the rate constant for exponential decay, which is derived in the context of a system \\ at equilibrium but is generally measured using a single cohort of individuals.
}

\section{Keywords}

quantitative reasoning, calculus, differential equations, math education, exponential function

\section{Creative Commons License}

(c) (1) (8)

This work is licensed under a Creative Commons Attribution-Noncommercial 4.0 License

\section{Cover Page Footnote}

Dorothy Wallace is a professor of mathematics at Dartmouth. She was 2000 New Hampshire CASE Professor of the Year, and the lead PI of the seminal NSF project, Mathematics Across the Curriculum. She recently finished a text in mathematical biology for first-year students, "Situated Complexity." She was a charter board member of the National Numeracy Network and is a founding co-editor of this journal. 


\section{Parts Of The Whole}

\section{A Column by D. Wallace}

The problem of how best to improve the numeracy of a society is a thorny one, embracing the learning process of a single student but rising in scale to include the management and alteration of an entire system of education. With the issue of quantitative literacy always in mind, this column considers various aspects of the systemic workings of education, the forces acting on classrooms, teachers and students, and mechanisms of both stasis and change. With the last issue, the column has pivoted to thoughts from developing and teaching "Math 4: Applications of Calculus to Mathematics and Biology," which Dartmouth biology students can take as an alternative to second-semester calculus (see Rheinlander and Wallace 2011, http://dx.doi.org/10.5038/1936-4660.4.1.3 in this journal).

\section{Teaching Quantitative Reasoning in an Exponential Decay Model}

Most introductory calculus courses include a discussion of the derivative and integral of the exponential function, with standard applications of exponential growth and decay. In particular, radioactive decay is presented in homework problems as a vehicle for understanding the concept of half-life, both in physical and mathematical terms. The exponential function is also the solution of the first differential equation most students see: $F^{\prime}=-a F$. Beginning calculus students can easily verify that any function of the form $F(t)=c e^{-a t}$ solves this equation, although texts and instructors often race past the conceptual difficulty of an equation whose solution is a function of another variable.

The half-life, $T_{1 / 2}$, of an exponentially decreasing function is defined as the elapsed time at which half of the original quantity remains. Most calculus students are shown the derivation of the relationship between the parameter " $a$ " and the half-life: $T_{1 / 2}=\ln 2 / a$, or equivalently $a=\ln 2 / T_{1 / 2}$. But as is often the case with straightforward calculus problems, when the example is moved from the physical to the biological sciences, the meaning behind the constants, the methods of measuring them, and the assumptions behind them all change. Making sense of them requires a level of quantitative reasoning beyond what is usually required in a calculus class and is an excellent excuse for strengthening the relationship between science and math through messy quantitative considerations. To quote an early mentor of mine, "Confusion is the first step in learning." Here we will return to that first step. 


\section{A Model for Insect Maturation}

Insect life cycles include a larval stage. The population dynamics of insects must take this phenomenon into account, because the habitat, carrying capacity, predation rates, and crowding effects for larvae may be completely different for adult insects. Mosquito larvae, for example, are aquatic, and the amount of time spent in the aquatic stages differs among species of mosquito. One little piece of the puzzle of modeling mosquito population dynamics is finding a reasonable expression of the rate at which larvae progress to adults.

Suppose we have a model of these population dynamics that is restricted by a carrying capacity and arrives at some equilibrium. At that equilibrium there will be a constant number of larvae present. It is reasonable to suppose that some of them are young larvae while others are about to mature, with all ages in between present. We could reasonably expect that a constant fraction of these larvae will mature to adults per unit time. That emerging fraction must be constant because we are thinking about a system at equilibrium. If $L$ is the larvae population, then per unit time some fraction of $L$, namely " $a L$," will leave that population to become adults. So, just looking at that one process, we will have an equation of the form $L^{\prime}=-a L$ describing maturation of larvae into adults. The parameter " $a$ " describes the relative rate (as percent of the population per unit time) at which this process is happening. In fact, most insect dynamics models that include larval stages use a term like this one to describe maturation. Of course, graphing the solution to such an equation produces a decreasing exponential function.

\section{Measuring Maturation}

It is not possible to measure the maturation "rate" directly. What biologists can measure directly is the maturation "time," or the number of days spent in the larval stage before emergence into an adult. Usually a cohort of recently laid eggs is placed in water (in the case of mosquitoes), fed, and observed until all the resulting larvae mature into adults. The biologists create a situation that is as unlike equilibrium as it could possibly be, where a single cohort passes more or less uniformly through all larval stages. One reason for studying the maturation time this way is that when multiple stages of larvae are present in the same habitat, the larger ones tend to eat the smaller ones. With a uniform cohort, all the larvae are close to the same size and this larval cannibalism (e.g., Reisen and Emory 1976; Church and Sherratt 1996) can be ignored.

From such a study, maturation times might be reported in one of two ways. All the individual times could be averaged to give a mean maturation time. Alternatively, the median maturation time might be reported, which is the time at which exactly half of the larvae have matured. 


\section{Finding a Maturation Rate}

The first problem with converting the maturation time into a maturation rate is that, technically, maturation times are measured for individuals. But, the notion of a maturation rate makes no sense for individuals. It is a description of a population, some of which mature per unit time. So, when estimating a maturation rate, one has to work from population parameters, such as those described by the mean and median maturation times.

Let's say the mean maturation time is 10 days. In a population at equilibrium and ignoring a possible death rate, one would expect one-tenth of the larvae to mature each day. This would make the parameter " $a$ " equal to 0.10 , or $10 \%$ per day.

On the other hand, if a median maturation time of 10 days is reported, we could take that number to be the time at which half the population has matured, or the half-life of maturation. This would make the parameter " $a$ " equal to $\ln (2) / 10=0.069$, or $6.9 \%$ per day. Other things being equal, for many models a slower maturation time will result in fewer adults per unit of carrying capacity. The difference between these two constants is therefore important.

\section{Implementing the Model}

Figure 1 shows the results of numerically solving the equation $F^{\prime}=-a F$ for both choices $(a=0.1$ and $a=0.069)$.

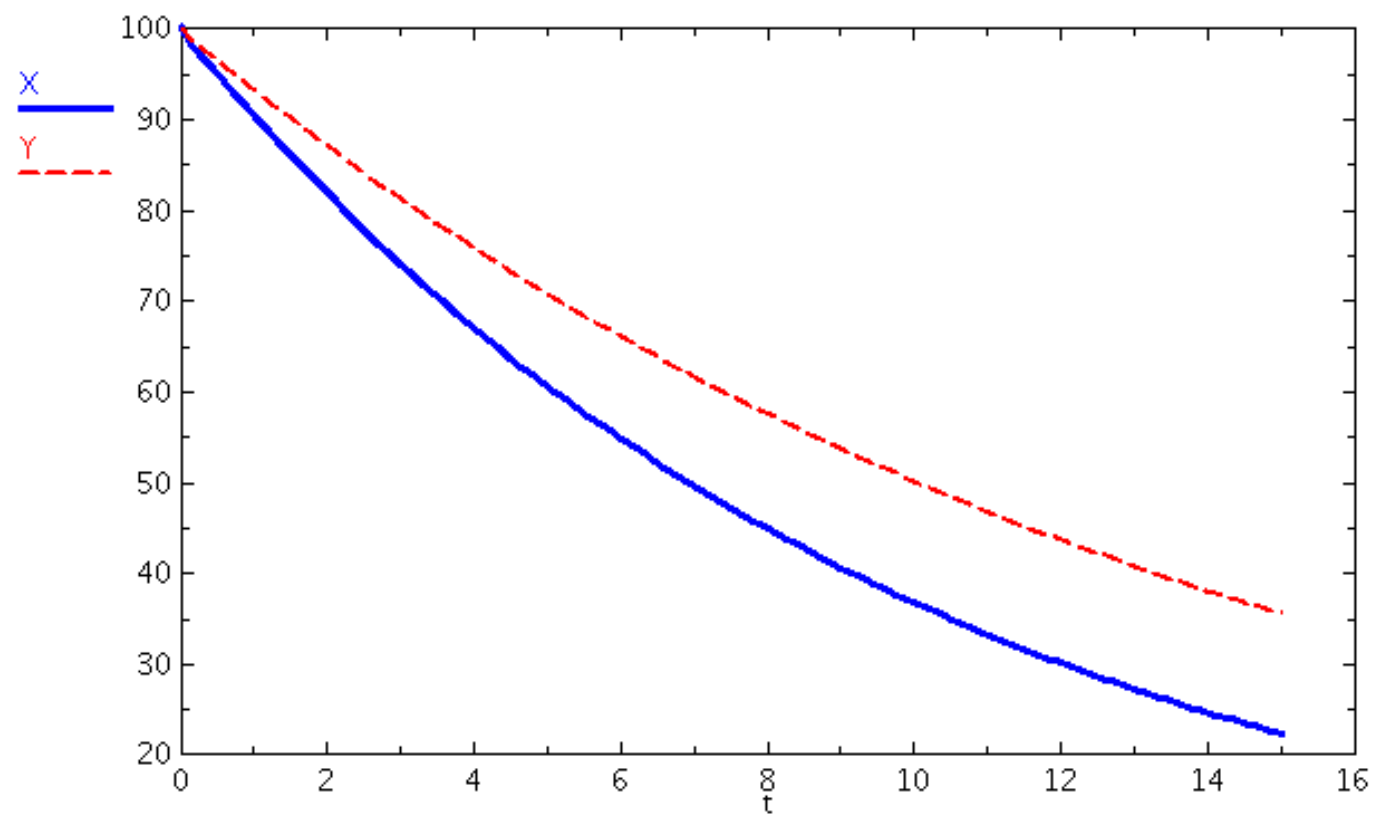

Figure 1. The dashed line shows the solution of $X^{\prime}=-0.069 X$ and the solid line shows the solution of $Y^{\prime}=-0.1 Y$. Both start at 100 . 
The dashed line in Figure 1 passes through the point $(10,50)$, reflecting the computation of half-life that resulted from using median maturation time. However something is not quite right in both of these graphs. As an example, the dashed line indicates that at day 2 over $12 \%$ of the larvae have already matured. This result seems unlikely if the maturation time is really closer to 10 days. Also, at day 14 the dashed line indicates that there are still around $37 \%$ of the larvae left. This result also seems strange if the maturation time is close to 10 days.

\section{Comparing the Model to Data}

What really happens with a single cohort? Biologists would observe a statistical distribution around the mean emergence time that has a characteristic spread of a few days on either side. In other words, no larvae would mature on days $1-8$, then many larvae would mature over the next few days, then no further maturation would occur because all of them would be adults at that point (or dead, which is another story). The greatest rate of maturation would be around day 10 .

In the exponential model above, the greatest rate of maturation is at the start, when there are many larvae. That observation follows from the original equation: $F^{\prime}=-a F$. Maturation is faster when $F$ is larger, which for a single cohort is at the beginning of the process.

Does this analysis mean we should throw out the model entirely? No, because it is based on reasonable assumptions for large mixed populations. On the other hand, we might want to make it work for a single cohort. While it is not true that maturation is strictly proportional to the whole population, it is probably true for the days that are near the measured mean maturation time. That is, $F^{\prime}=-a F$ is probably a good description of how days 8-12 work.

\section{Exponential Decay, Extended}

One way to handle this subtlety is to break the process into pieces. Conveniently, larvae pass through distinguishable stages. Mosquitoes have six juvenile stages: egg; four "instars," which are aquatic; and the pupa, which floats until the adult mosquito emerges. The 10 days might be broken into these six parts: 1 day mean egg hatching time, 2 days mean time in each instar, 1 day mean time as pupa. Moving maturing larvae from one stage to the next, we would get a sevencompartment model that looks like this (ignoring birth, death, and other factors):

$$
\begin{aligned}
& E^{\prime}=-E \\
& L_{1}{ }^{\prime}=E-0.5 L_{1} \\
& L_{2}{ }^{\prime}=0.5 L_{1}-0.5 L_{2} \\
& L_{3}{ }^{\prime}=0.5 L_{2}-0.5 L_{3} \\
& L_{4}{ }^{\prime}=0.5 L_{3}-0.5 L_{4} \\
& P^{\prime}=0.5 L_{4}-P \\
& A^{\prime}=P
\end{aligned}
$$


When the system of equations is solved numerically we can see something a bit more reasonable, as in Figure 2. It is worth mentioning that a system like this will make sense to calculus students who already understand the first equation in it. It's just a question of asking where eggs go when they mature, and what happens after that? One aspect of numeracy in calculus is being able to take a relatively simple relationship and see how it can be used in different contexts, or in this case repeatedly, to describe something relatively complex.

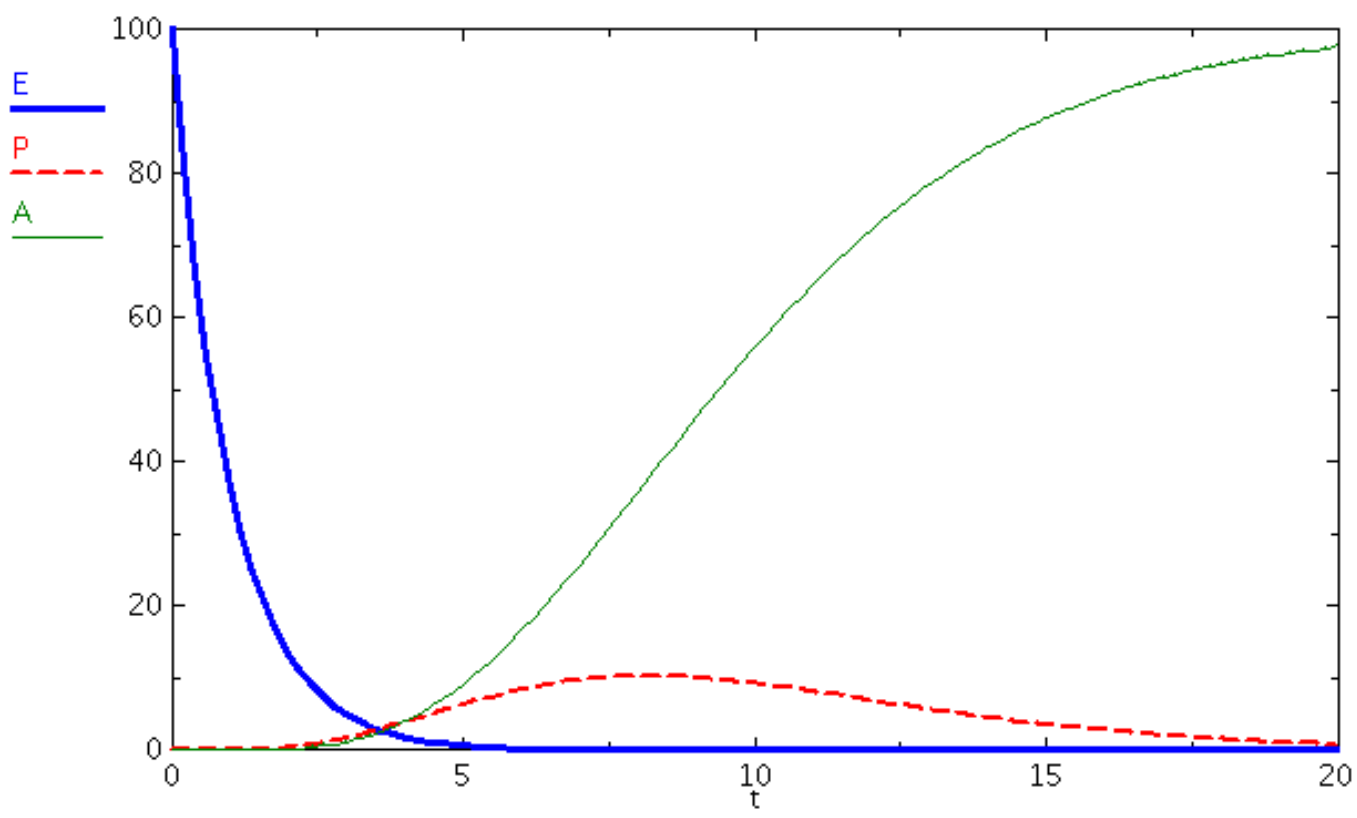

Figure 2. Numerical solution to the seven compartment system described above. The decreasing solid line is the number of eggs declining as they mature into $L_{I}$ (not pictured). The dashed line is pupa, rising as $L_{4}$ (not pictured) matures into $P$, and then declining as $P$ matures into $A$.

At day 10, Figure 2 shows that approximately half of all juveniles are now adult. But it behaves more like the data, as almost no adults appear before day 5, and nearly all of them are present at day 20 . The spread is probably still too large, and yet it is a big improvement on the model in Figure 1. In particular, the fastest rate of maturation into adulthood (the slope of the rising solid line) is around day 10 (a little early, actually), as observed in a typical data set (Reisen, Mahmood and Parveen 1979). One could achieve a better match to a particular data set by adjusting the rate constants and adding more compartments.

\section{Richness of Context Leads to Depth of Numeracy}

In the sciences, quantitative reasoning plays multiple roles. It helps develop the common sense principles behind a model. It questions how parameters are derived 
from measurements. It compares model output to data. It constantly asks, "Does this model make sense?" But to see all of these useful roles in action requires investigating situations where parameters are not just given, but derived from measurements. It requires enough complexity that the relationship of common sense assumptions to model output is not straightforward.

Increasingly, critical thinking about quantitative science requires the use of tools for numerical calculation. The difference between the answers given by models in Figures 1 and 2 could be derived theoretically, because these are linear models. It would be a tedious task! Ultimately though, it is far more satisfying to look at the numerical output and think about it visually. Graphical interpretation is something everyone agrees is part of numeracy, and computational tools let us state complicated questions in a visual form.

Returning to the comment of my friend Herb, who said, "Confusion is the first step in learning," it is possible to recast quantitative reasoning in his terms. Quantitative reasoning could be defined as the process of becoming un-confused about numbers. To practice this process, it is necessary for the learner to start in a confused state. And, especially in the sciences, it is best if the confusion arises from the natural complexity of a good problem.

\section{References}

Church, Stuart C., and Thomas N. Sherratt. 1996. "The Selective Advantages of Cannibalism in a Neotropical Mosquito." Behavioral Ecology and Sociobiology 39 (2): 117-123. http://dx.doi.org/10.1007/s002650050273.

Reisen, Willaim K., and Richard W. Emory. 1976. "Cannibalism in Anopheles stephensi Liston.” Mosquito News 36: 198-200.

Reisen, William K., Farida Mahmood, and Tauheeda Parveen. 1979. "Laboratory observations on the time of mating of Anopheles culicifacies Giles."

Mosquito News 39: 328-333. 